\title{
Piwil2 modulates the invasion and metastasis of prostate cancer by regulating the expression of matrix metalloproteinase- 9 and epithelial-mesenchymal transitions
}

\author{
YANFENG YANG, XUEPEI ZHANG, DONGKUI SONG and JINXING WEI \\ Department of Urology, The First Affiliated Hospital of Zhengzhou University, \\ Zhengzhou, Henan 450000, P.R. China
}

Received April 14, 2014; Accepted January 23, 2015

DOI: $10.3892 / \mathrm{ol} .2015 .3392$

\begin{abstract}
Piwi-like RNA-mediated gene silencing 2 (Piwil2) is an oncogene that is highly expressed in breast, gastric, colorectal and papillary thyroid cancer. As a candidate oncogene, its role in prostrate cancer has yet to be elucidated. In the present study, 30 tumor specimens and four prostate cancer cell lines were analyzed. The total RNA and protein from the specimens and the cells were analyzed using quantitative polymerase chain reaction and western blotting, respectively. The expression of Piwil2 in PC-3 cells was knocked down using specific small hairpin RNA. Transwell assays and wound-healing models were used to assess cell invasion and migration. In addition, the expression of several factors associated with epithelial-mesenchymal transitions (EMT) were evaluated by western blotting. The results revealed that the Piwil2 gene was associated with the Gleason score and the tumor-node-metastasis stage of the tumor tissues. Cell invasion and migration decreased significantly in PC-3 cells with knocked-down Piwil2. In addition, silencing Piwil2 downregulated the expression of $\mathrm{N}$-cadherin, Twist and vimentin and upregulated the expression of E-cadherin, factors associated with EMT, and also reduced the expression of matrix metalloproteinase-9. Piwil2 was demonstrated to possess an important role in the invasive ability of prostate cancer, and therefore, may be a potential therapeutic target for the treatment of this cancer.
\end{abstract}

\section{Introduction}

Prostate cancer has become one of the most common types of cancer, and is currently the second most lethal disease affecting the elderly male population in developed countries. In 2010 and 2013, prostrate cancer led to 80,900 and

Correspondence to: Dr Jinxing Wei, Department of Urology, The First Affiliated Hospital of Zhengzhou University, 1 Jianshe Road, Zhengzhou, Henan 450000, P.R. China

E-mail: weijingxing2001@outlook.com

Key words: Piwil2, prostate cancer, invasion, metastasis
33,720 mortalities, respectively (1-3). Although progress has been made in the treatment of prostate cancer, the majority of patients succumb to tumor metastasis. Furthermore, tumors recur in $\sim 30 \%$ of patients within 12-18 months of undergoing a prostatectomy $(4,5)$. Therefore, an improved understanding of the molecular mechanisms involved in the pathogenesis of prostate cancer, including those underlying metastasis and invasion, is urgently required.

The Piwi genes, which were identified a number of decades ago, were the first class of genes known to be required for stem cell self-renewal in a diverse range of organisms $(6,7)$. Piwi-like RNA-mediated gene silencing 2 (Piwil2) belongs to the Ago/Piwi family, which is comprised of Piwill/Hiwi, Piwil2/Hili, Piwil3 and Piwil4/Hiwi2. The human-derived Piwil2 gene regulates RNA silencing and transcription, functions in spermatogenesis, and is involved in the self-renewal and differentiation of normal testis and fetal tissues $(8,9)$. Piwil2, as a candidate oncogene in previous studies, is highly expressed in breast, gastric, colorectal and papillary thyroid cancer, and is closely associated with the occurrence and progression of tumors (10-14).

The association between Piwil2 and prostate cancer is rarely reported. The present study therefore aimed to investigate this association and discern the potential underlying molecular mechanisms.

\section{Materials and methods}

Cell lines and primary tumor specimens. The PC-3, 22RV1, DU-145 and LNCaP cell lines (American Type Culture Collection, Manassas, VA, USA) were cultured in RPMI-1640 complete medium (GE Healthcare Life Sciences, Logan, UT, USA) containing $10 \%$ fetal calf serum, 100 units $/ \mathrm{ml}$ penicillin and $100 \mu \mathrm{g} / \mathrm{ml}$ streptomycin. The immortalized normal prostate epithelial cell line RWPE-1 was cultured in keratinocyte serum-free medium containing $0.05 \mathrm{mg} / \mathrm{ml}$ bovine pituitary extract and $5 \mathrm{ng} / \mathrm{ml}$ recombinant epidermal growth factor (Gibco Life Technologies, Carlsbad, CA, USA). The cell lines were cultivated in a $37^{\circ} \mathrm{C}$ incubator with $5 \%$ $\mathrm{CO}_{2}$. Next, the cells were digested with $0.25 \%$ trypsin every three to four days for passage. All cells used in the experiments were in the logarithmic growth phase. 
In total, 30 tumor specimens were obtained from patients with prostate cancer at The First Affiliated Hospital of Zhengzhou University (Zhengzhou, China). The patients, who were aged between 45 and 89 years old, underwent a radical prostatectomy, without receiving any other treatments, between July and November 2012. The present study was approved by the Ethics Committee of The First Affiliated Hospital of Zhengzhou University. Informed consent was obtained from each patient, conforming to the tenets of the Declaration of Helsinki (15).

Total RNA extract and reverse transcription-quantitative polymerase chain reaction ( $R T-q P C R)$. Human tumor tissues and pericarcinomatous tissues obtained from patients with prostate cancer were immediately frozen in liquid nitrogen and stored at $-80^{\circ} \mathrm{C}$. The total RNA from the tumor tissues, pericarcinomatous tissues and all aforementioned cell lines were extracted using a total RNA extraction kit, according to the manufacturer's instructions (Invitrogen Life Technologies, Carlsbad, CA, USA). Next, 100 ng total RNA was used for qPCR, according to the instructions of the SYBR Green PCR master mix kit (Invitrogen Life Technologies). Each amplification was performed using one of the following primers: $\beta$-actin forward, 5'-CACCCA GCACAATGAAGAT-3' and reverse, 5'-CAAATAAAGCCT GCCAAT-3'; Piwil2 forward, 5'-TCATGGGGCCATCAGAAG-3' and reverse, 5'-CCATCCCGATCACCATTAAC-3'; matrix metalloproteinase (MMP)-2 forward, 5'-GATACCCCT TGACGGTAAGG-3' and reverse, 5'-CCTTCTCCCAAGGTC CATAGC-3'; MMP-9 forward, 5'-GGGACGCAGACATCGTCATC-3' and reverse, 5'-TCGTCATCGTCGAAATGGGC-3'; E-cadherin forward, 5'-TGCTCTTCCAGGAACCTCTGTG-3' and reverse, 5'-GGTGACCACACTGATGACTCCTG-3'; N-cadherin forward, 5'-GGTGGAGGAGAAGAAGACCAG-3' and reverse, 5'-GGCATCAGGCTCCACAGT-3'; vimentin forward, 5'-GGGACCTCTACGAGGAGGAG-3' and reverse, 5'-CGCATTGTCAACATCCTGTC-3'; and Twist forward, 5'-GCTGTGCTTACTCTAGCCATC-3' and reverse, 5'-TGA GGCATTTGCTCACATCAC-3'. All PCR experiments were performed in triplicate using the Bio-Rad C1000 Touch $^{\mathrm{TM}}$ Thermal Cycler (Bio-Rad Laboratories, Inc., Hercules, CA, USA). PCR was performed under the following conditions: Denaturation at $93^{\circ} \mathrm{C}$ for $2 \mathrm{~min}$, followed by 40 cycles of $93^{\circ} \mathrm{C}$ for $1 \mathrm{~min}, 55^{\circ} \mathrm{C}$ for $1 \mathrm{~min}$ and $72^{\circ} \mathrm{C}$ for $1 \mathrm{~min}$, and extension at $72^{\circ} \mathrm{C}$ for $7 \mathrm{~min}$.

Western blot analysis. The cells were lysed at $2-8^{\circ} \mathrm{C}$ in RIPA solution containing $1 \%$ proteasome inhibitor (Beyotime Institute of Biotechnology, Jiangsu, China), for $30 \mathrm{~min}$. The total protein concentration was then determined using the Bradford method. In total, $30 \mu \mathrm{g}$ of denaturized total protein was loaded into each lane for SDS-PAGE. The bands were then transferred to polyvinylidene fluoride membranes (EMD Millipore, Billerica, MA, USA). The membranes were incubated with blocking buffer (Sigma-Aldrich, St. Louis, MO, USA) containing 5\% bovine serum albumin in phosphate-buffered saline for $2 \mathrm{~h}$ at room temperature. Incubation was then performed using the primary monoclonal mouse anti-human Piwil2 (dilution, 1:800; cat. no. LS-C62097-100; LifeSpan BioSciences, Inc., Seattle, WA, USA), monoclonal mouse anti-human E-cadherin (dilution, 1:800; cat. no. 5296S; Cell Signaling Technology, Inc., Danvers, MA, USA), monoclonal mouse anti-human
N-cadherin (dilution, 1:500; cat. no. 14215S; Cell Signaling Technology, Inc.), polyclonal rabbit anti-human Twist (dilution, 1:800; cat. no. ab50581; Abcam, Cambridge, UK), monoclonal mouse anti-rabbit vimentin (dilution, 1:800; cat. no. 9775S; Cell Signaling Technology, Inc.) and polyclonal mouse anti-human $\beta$-actin (dilution, 1:1,500; cat. no. sc-7210; Santa Cruz Biotechnology, Inc., Dallas, TX, USA) antibodies overnight at $4^{\circ} \mathrm{C}$. The membranes were subsequently incubated with horseradish peroxidase-conjugated polyclonal rabbit anti-mouse (dilution, 1:4,000; cat. no. A0216; Beyotime Institute of Biotechnology) or polyclonal goat anti-rabbit (dilution, 1:4,000; cat. no. A0239; Beyotime Institute of Biotechnology) secondary antibodies for $2 \mathrm{~h}$ at room temperature. Detection was performed using the enhanced chemiluminescent substrate (Bio-Rad Laboratories, Inc.).

Knockdown of the Piwil2 gene using short hairpin RNA (shRNA). A recombinant lentivirus containing a green fluorescent protein (GFP) reporter and a sequence encoding a Piwil2-specific shRNA (shRNA group) or a scramble shRNA (mock group) was synthesized by Shanghai Rainbow Chemistry Co., Ltd. (Shanghai, China). The sequences of the Piwil2 and scrambled shRNAwere 5'-AAACCTTTGGACCCAGCTCTG-3' and 5'-GTACCGCACGTCATTCGTATC-3', respectively (14). The PC-3 cells were transfected with viral supernatants according to the manufacturer's instructions. In total, $5 \times 10^{5}$ cells were washed three times prior to the cell transfection, and $20 \mathrm{pmol}$ shRNA, with $3 \mu \mathrm{g} / \mathrm{ml}$ polybrene in serum-free medium, was added to the cells and incubated at $37^{\circ} \mathrm{C}$. Fresh culture medium was added after $6 \mathrm{~h}$. The cells expressing GFP were sorted using a flow cytometer (FACS AriaTM II; BD Biosciences, Franklin Lakes, NJ, USA) when the cells had reached $70-80 \%$ confluence. The silencing effect on the Piwil2 gene was assessed by qPCR and western blot analysis, according to the aforementioned protocols.

Tumor invasion and migration. A Transwell unit was used to investigate cellular invasion. The units were placed in 24-well plates and the upper chamber was coated with Matrigel (BD Biosciences). In total, $200 \mathrm{ml}$ of cell solution, at a density of $5 \times 10^{4}$ cells $/ \mathrm{ml}$, resuspended in serum-free medium, was added to the upper chamber, and medium containing $10 \%$ fetal bovine serum was added to the lower chamber. Subsequent to a 24-h incubation, the cells that had passed through the membrane were fixed with $4 \%$ paraformaldehyde for $15 \mathrm{~min}$, and then stained with crystal violet. The cells in five random fields were counted under an Olympus CX41 light microscope (magnification, x200; Olympus Corporation, Shanghai, China). In addition, a wound-healing assay was performed in order to measure the extent of cell migration. In total, $1 \times 10^{4} \mathrm{PC}-3$ cells were transplanted into a 24-well plate. When the cells had grown to cover the entire bottom of the plate well, a straight-line scratch was made using a $20-\mu 1$ pipette tip. Subsequent to a 48 -h incubation period in the serum-free medium, images of the cells were captured using an Olympus CKX31 reverse-phase microscope (Olympus Corporation).

Statistical analysis. The data were analyzed using SPSS software version 15.0 (SPSS, Inc., Chicago, IL, USA). The data derived from the tumor invasion assay of the shRNA and 

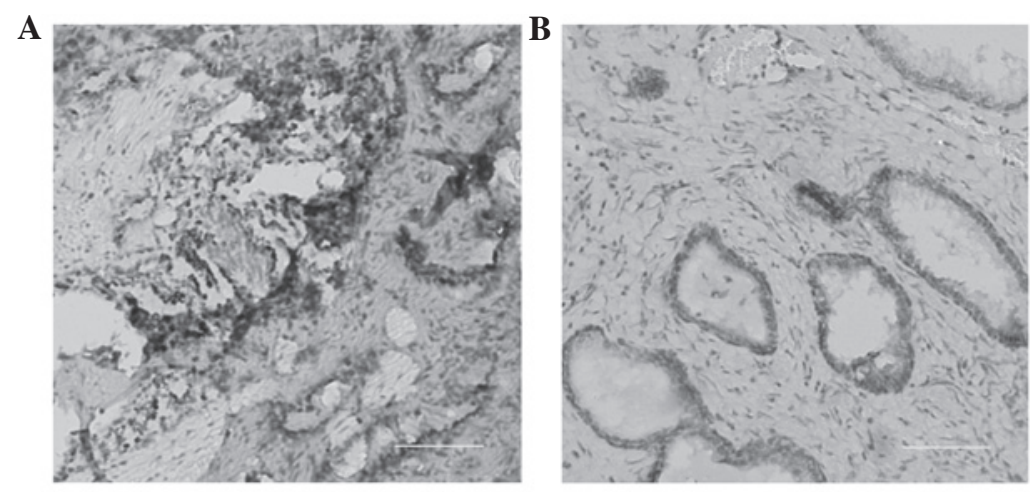

Figure 1. Immunohistochemical analysis revealing the expression of the Piwi-like protein 2 (Piwil2). Piwil2 is highly expressed in (A) prostate cancer cells compared with (B) adjacent normal tissue. Scale bar, $200 \mu \mathrm{m}$.

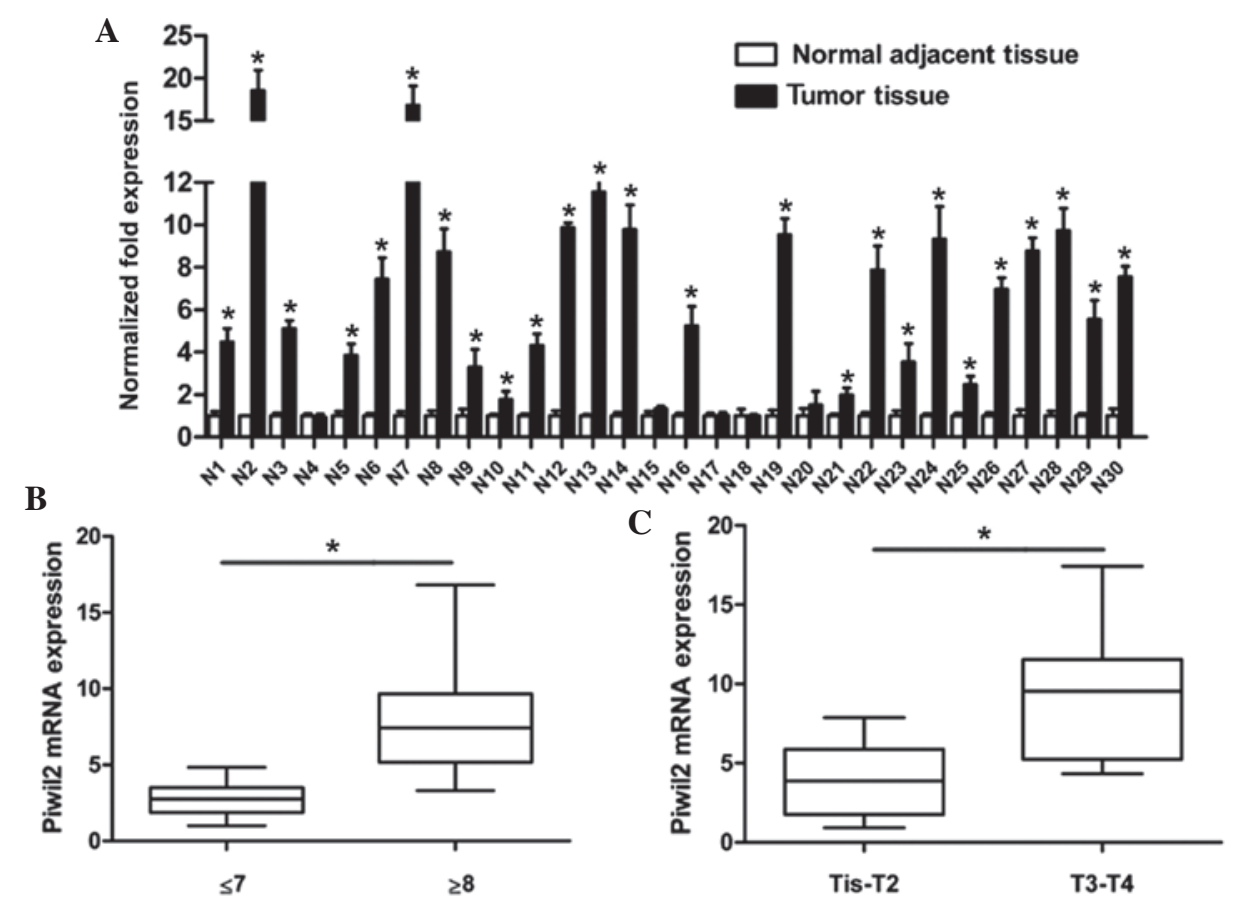

Figure 2. Results of quantitative polymerase chain reaction revealing the expression of the Piwil2 gene in 30 patients. (A) Compared with adjacent normal tissues, the expression of the Piwil2 gene in prostate cancer tissues was significantly higher. The expression of the Piwil2 gene was also significantly higher in patients with (B) a high Gleason score ( $\geq 8)$ and (C) an advanced tumor-node-metastasis stage (T3-T4). ${ }^{*} \mathrm{P}<0.05$.

mock groups were assessed using a $t$-test for two independent samples or the rank-sum test for non-normal distribution, and the data are presented as the mean \pm standard deviation. A value of $\mathrm{P}<0.05$ was considered to indicate a statistically significant difference.

\section{Results}

Expression of Piwil2 in clinically obtained specimens of prostate cancer. In order to investigate the expression of the Piwil2 gene and protein in patients with different histological grades of prostate cancer, RT-qPCR and western blot analysis was performed. Immunohistochemical staining revealed a high expression of Piwi-like protein 2 in prostate cancer cells (Fig. 1A) when compared with adjacent normal tissue (Fig. 1B). The results of RT-qPCR revealed that $83.33 \%$ of tumor tissues (25/30) possessed a higher level of Piwil2 than the associated adjacent tissues (Fig. 2A). In addition, Piwil2 expression was positively associated with the Gleason score $(\mathrm{P}=0.002)$ and the tumor-node-metastasis $(\mathrm{TNM})$ stage $(\mathrm{P}=0.003)$ of the tumor tissue. As shown in Fig. 2B and C, Piwil2 was overexpressed in patients with a high Gleason score $(\geq 8)$ and an advanced TNM stage (T3-T4).

Expression of Piwil2 in prostate cancer cell lines. RT-qPCR was performed in order to detect the level of Piwil2 in the normal prostate RWPE-1 cell line and in the PC-3, 22RV1, DU-145 and $\mathrm{LNCaP}$ prostate cancer cell lines. The level of Piwil 2 in the tumor cell lines was higher compared with the normal prostate cell line (Fig. 3A). Of the four prostate cancer cell lines analyzed, Piwil2 expression was highest in the PC-3 cells.

Subsequent to transfection with shRNA or mock recombinant lentiviruses, GFP-positive PC-3 cells were collected by flow cytometry, sorted and then cultured (Fig. 3B). The 
A

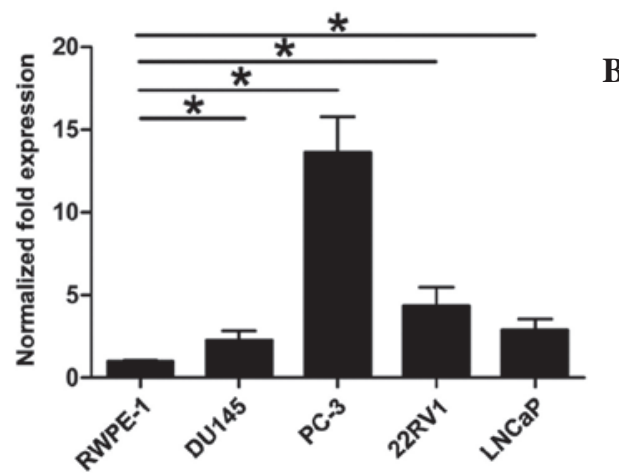

B
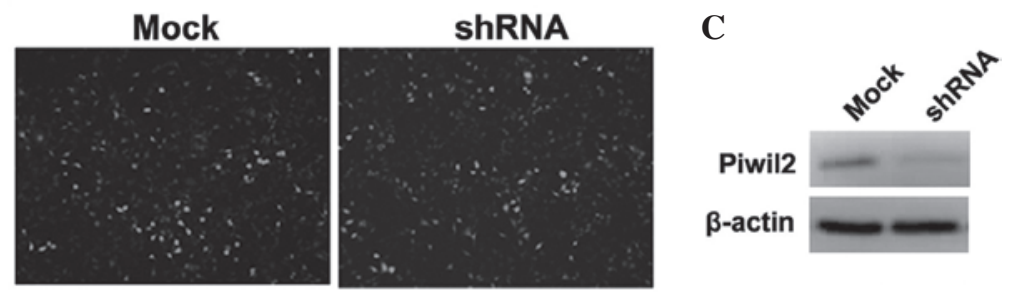

Figure 3. Knock-down of the Piwil2 gene in the prostate cancer cell lines using specific small hairpin RNA. (A) Compared with the normal RWPE-1 prostate epithelial cell line, an overexpression of Piwil2 mRNA was evident in the PC-3, 22RV1, DU-145 and LNCaP prostate cancer cell lines. (B) Piwil2-targeted shRNA (shRNA group) and scrambled shRNA (mock group) were successfully transfected into PC-3 cells. (C) Western blot analysis revealing that the expression of the Piwil2 gene decreased in the shRNA group. " $\mathrm{P}<0.05$.

A

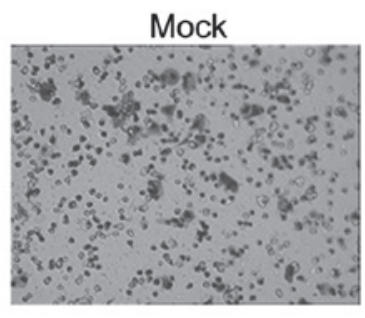

C

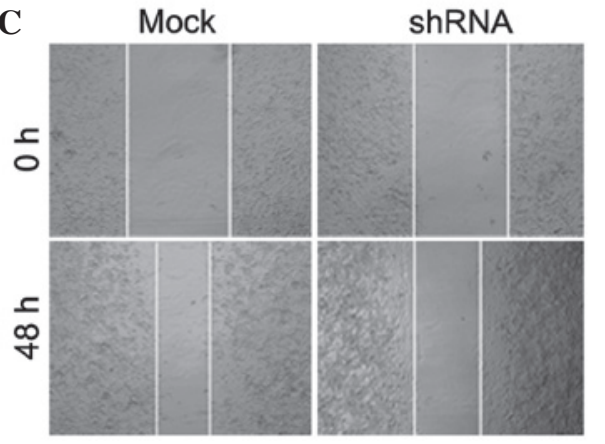

ShRNA
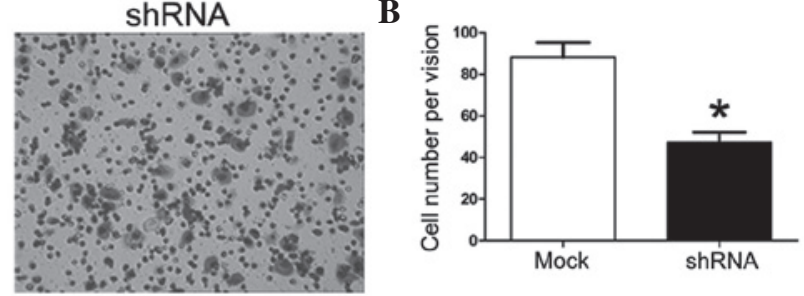

D

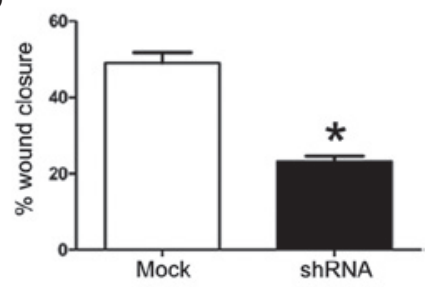

Figure 4. Effect of Piwil2 knock-down on tumor invasion and migration in vitro. (A) A Transwell assay was used to evalute cellular invasion in the Piwil2-targeted small hairpin (sh)RNA group and the mock group. (B) The number of cells in the shRNA group was significantly lower than in the mock group. Knock-down of the Piwil2 gene decreased (C) cell migration and (D) wound closure in the Piwil2-targeted shRNA group. ${ }^{*} \mathrm{P}<0.05$.

A

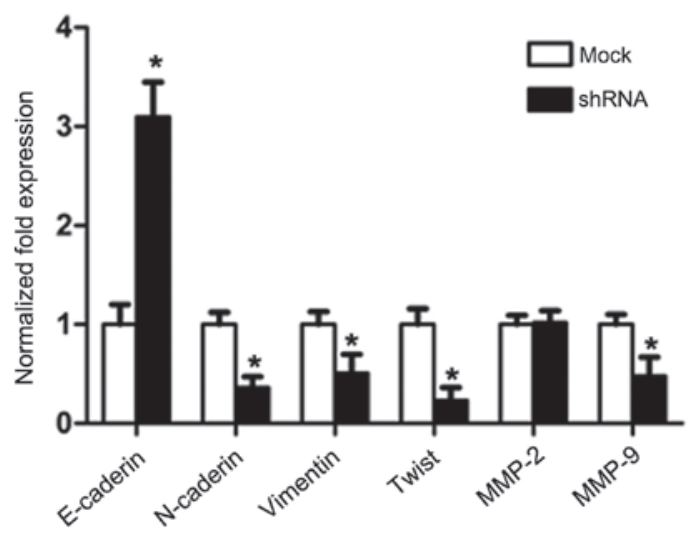

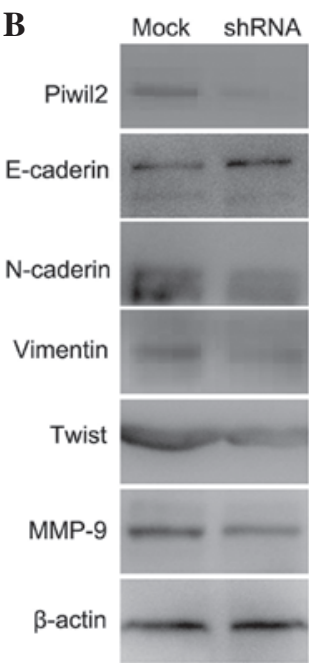

Figure 5. Knock-down of Piwil2 in PC-3 cells reduced matrix metalloproteinase (MMP)-9 expression and inhibited epithelial-mesenchymal transitions. The mRNA and protein expression of E-cadherin, N-caderin, vimentin, twist, MMP-2 and -9 were detected by (A) reverse transcription-quantitative polymerase chain reaction and (B) western blotting, respectively. ${ }^{*} \mathrm{P}<0.05$. 
silencing effect on the Piwil2 gene was confirmed by western blot analysis. Compared with the mock group, the Piwil2 gene was significantly downregulated in cells of the shRNA group (Fig. 3C). This demonstrated that the lentivirus-encoding Piwil2-targeted shRNA effectively decreased Piwil2 expression in the PC-3 cells.

Function of Piwil2 in invasion and migration in vitro. For the tumor invasion assay, the number of cells on the lower chamber of the Transwell unit was counted. The number of cells in the shRNA group (49.19 \pm 9.09$)$ was significantly lower compared with the mock group $(90.43 \pm 14.54)(\mathrm{P}=0.000$; Fig. 4A and B). A wound-healing assay was also performed in order to measure the distance of cell migration in serum-free medium. The difference in the width of the scratch between 0 and $48 \mathrm{~h}$ was processed using Image 1.37 software (National Institutes of Health, Bethesda, MA, USA). Knockdown of the Piwil2 gene significantly decreased cell migration in the shRNA group (Fig. 4C and D). The extent of wound closure in the mock group $(49.64 \pm 5.63)$ was significantly higher compared with the shRNA group $(23.53 \pm 2.66)(\mathrm{P}=0.000)$. These results indicate that knock-down of the Piwil2 gene in the PC-3 cells decreased invasion and migration in vitro.

Knockdown of Piwil2 decreases the expression of MMP-2/9 and inhibits epithelial-mesenchymal transitions (EMT). As MMPs and EMT have important roles in tumor invasion and metastasis, the levels of MMP-2 and -9, together with the expression of the primary biomarkers of EMT, E-cadherin, N-cadherin, vimentin and Twist, were evaluated by RT-qPCR and western blotting. The results revealed that the expression of vimentin, Twist and MMP-9 in the PC-3 cells of the shRNA group was downregulated, but that E-cadherin was upregulated and MMP-2 exhibited no change in expression (Fig. 5A and B).

\section{Discussion}

The human-derived Piwil2 gene was first identified as a stem and testis cell-specific gene (7). As tumor cells and stem cells have the capacity of self-renewal, it is likely that their regulatory mechanisms are similar. Piwil2 is also expressed in several aforementioned tumors. The Piwil2 gene has been highly conserved during evolution and serves as a key factor in maintaining the self-renewal and differentiation of testis and embryonic cells in normal tissue. In addition, Piwil2 has an important role in the development, differentiation and regulation of precancerous stem cells (9). An overexpression of Piwil2 can disturb cell division, and therefore lead to malignant transformation. Piwil proteins have been used as predictive markers for a number of cancers $(12,16)$, particularly for the early detection of disease (6). Furthermore, an overexpression of Piwil2 has been associated with a higher tumor stage and a poorer prognosis $(17,18)$. Despite this, the role of Piwil2 in prostate cancer has been investigated in relatively few studies $(6,19)$.

The present study demonstrated that the Piwil2 gene is highly expressed in surgical tissues and prostate cancer cell lines. Furthermore, it revealed that the level of Piwil2 was positively associated with the Gleason score and TNM stage of the tumor. These results indicate that Piwil2 has a positive role in the development and progression of prostate cancer. Out of the four prostate cancer cell lines that were analyzed, PC-3 cells possess the capacity of high invasion and were derived from bone metastasis, which accounts for up to $90 \%$ of all metastatic types of prostate cancer (20). A recombinant lentivirus encoding Piwil2-targeted-shRNA was therefore used to knock down Piwil2 expression in PC-3 cells. As predicted, the results revealed that the inhibition of Piwil2 significantly reduced the invasion and metastasis of PC-3 cells.

A previous study identified that Piwil 2 modulated colon cancer metastasis via the regulation of MMP-9 transcriptional activity (14). A further study revealed that MMP-9 in PC-3 cells induced the activity of osteoclasts, and enhanced the invasion of bone metastasis (21). In order to elucidate the potential underlying mechanisms, the present study analyzed the expression of MMPs and consequently identified a downregulation in the expression of MMP-9 in the Piwil2-targeted shRNA PC-3 cells. EMT has gained interest for its involvement in the early steps of invasion and metastasis in malignant tumors of epithelial origin (22). Whether or not Piwil2 activates the process of EMT has yet to be elucidated. Therefore, the present study evaluated the expression of the primary biomarkers of EMT using western blotting and RT-qPCR in the Piwil2-targeted shRNA PC-3 cells. The epithelial marker, E-cadherin, was upregulated, whereas the associated transcription factors involved in EMT, namely vimentin, N-cadherin and Twist, were downregulated.

In summary, the present study revealed that the expression of Piwil2 is positively correlated with the Gleason score and TNM stage of patients with prostate cancer. The Piwil2 gene is involved in the invasion and metastasis of tumor cells by regulating the levels of MMP-9 and modulating EMT. This suggests that Piwil2 participates in tumor aggressiveness and progression. Therefore, Piwil2 may be a novel therapeutic target in the treatment of prostate cancer.

\section{Acknowledgements}

This study was supported by grants from the National Natural Science Foundation of China (no. 81272823), and the Henan Science and Technology Research Foundation (no. 122102310047).

\section{References}

1. Jemal A, Siegel R, Ward E, et al: Cancer statistics, 2008. CA Cancer J Clin 58: 71-96, 2008.

2. Beltran H, Beer TM, Carducci MA, et al: New therapies for castration-resistant prostate cancer: efficacy and safety. Eur Urol 60: 279-290, 2011.

3. Siegel R, Naishadham D and Jemal A: Cancer statistics, 2013. CA Cancer J Clin 63: 11-30, 2013.

4. Chang SS and Kibel AS: The role of systemic cytotoxic therapy for prostate cancer. BJU Int 103: 8-17, 2009.

5. Ni J, Cozzi P, Hao J, et al: Epithelial cell adhesion molecule (EpCAM) is associated with prostate cancer metastasis and chemo/radioresistance via the PI3K/Akt/mTOR signaling pathway. Int J Biochem Cell Biol 45: 2736-2748, 2013.

6. Lee JH, Schütte D, Wulf G, et al: Stem-cell protein Piwil2 is widely expressed in tumors and inhibits apoptosis through activation of Stat3/Bcl-XL pathway. Hum Mol Genet 15: 201-211, 2006.

7. Cox DN, Chao A, Baker J, et al: A novel class of evolutionarily conserved genes defined by piwi are essential for stem cell self-renewal. Genes Dev 12: 3715-3727, 1998. 
8. Sasaki T, Shiohama A, Minoshima S and Shimizu N: Identification of eight members of the Argonaute family in the human genome. Genomics 82: 323-330, 2003.

9. Kuramochi-Miyagawa S, Kimura T, Ijiri TW, et al: Mili, a mammalian member of piwi family gene, is essential for spermatogenesis. Development 131: 839-849, 2004.

10. Liu X, Sun Y, Guo J, et al: Expression of hiwi gene in human gastric cancer was associated with proliferation of cancer cells. Int J Cancer 118: 1922-1929, 2006.

11. He G, Chen L, Ye Y, et al: Piwil2 expressed in various stages of cervical neoplasia is a potential complementary marker for p16. Am J Transl Res 2: 156-169, 2010.

12. Liu JJ, Shen R, Chen L, et al: Piwil2 is expressed in various stages of breast cancers and has the potential to be used as a novel biomarker. Int J Clin Exp Pathol 3: 328-337, 2010.

13. Yin DT, Li HQ, Wang YF, et al: Expression of Piwil2 and its relationship with tumor invasion and metastasis in papillary thyroid carcinoma. Zhonghua Er Bi Yan Hou Tou Jing Wai Ke Za Zhi 46: 237-239, 2011 (In Chinese).

14. Li D, Sun X, Yan D, et al: Piwil2 modulates the proliferation and metastasis of colon cancer via regulation of matrix metallopeptidase 9 transcriptional activity. Exp Biol Med (Maywood) 237: 1231-1240, 2012

15. General Assembly of the World Medical Association: World Medical Association Declaration of Helsinki: Ethical principles for medical research involving human subjects. J Am Coll Dent 81: $14-18,2014$
16. Wang Y, Liu Y, Shen X, et al: The PIWI protein acts as a predictive marker for human gastric cancer. Int J Clin Exp Pathol 5: 315-325, 2012.

17. Oh SJ, Kim SM, Kim YO and Chang HK: Clinicopathologic implications of PIWIL2 expression in colorectal cancer. Korean J Pathol 46: 318-323, 2012.

18. Greither T, Koser F, Kappler M, et al: Expression of human Piwi-like genes is associated with prognosis for soft tissue sarcoma patients. BMC Cancer 12: 272, 2012.

19. Shaikhibrahim Z, Lindstrot A, Ochsenfahrt J, Fuchs K and Wernert N: Epigenetics-related genes in prostate cancer: Expression profile in prostate cancer tissues, androgensensitive and -insensitive cell lines. Int J Mol Med 31: 21-25, 2013.

20. Bubendorf L, Schöpfer A, Wagner U, et al: Metastatic patterns of prostate cancer: an autopsy study of 1,589 patients. Hum Pathol 31: 578-583, 2000.

21. Dong Z, Bonfil RD, Chinni S, et al: Matrix metalloproteinase activity and osteoclasts in experimental prostate cancer bone metastasis tissue. Am J Pathol 166: 1173-1186, 2005.

22. Boyer B, Vallés AM and Edme N: Induction and regulation of epithelial-mesenchymal transitions. Biochem Pharmacol 60: 1091-1099, 2000. 